\title{
A cradle for new proteins: trigger factor at the ribosome Timm Maier ${ }^{1}$, Lars Ferbitz ${ }^{1}$, Elke Deuerling ${ }^{2}$ and Nenad Ban ${ }^{1}$
}

\begin{abstract}
Newly synthesized proteins leave the ribosome through a narrow tunnel in the large subunit. During ongoing synthesis, nascent protein chains are particularly sensitive to aggregation and degradation because they emerge from the ribosome in an unfolded state. In bacteria, the first protein to interact with nascent chains and facilitate their folding is the ribosomeassociated chaperone trigger factor. Recently, crystal structures of trigger factor and of its ribosome-binding domain in complex with the large ribosomal subunit revealed that the chaperone adopts an extended 'dragon-shaped' fold with a large hydrophobic cradle, which arches over the exit of the ribosomal tunnel and shields newly synthesized proteins. These structural results, together with recent biochemical data on trigger factor and its interplay with other chaperones and factors that interact with the nascent chain, provide a comprehensive view of the role of trigger factor during cotranslational protein folding.
\end{abstract}

\begin{abstract}
Addresses
${ }^{1}$ Institute for Molecular Biology and Biophysics, Swiss Federal Institute of Technology (ETH Zürich), Hoenggerberg, HPK Building, $\mathrm{CH}-8093$ Zürich, Switzerland

${ }^{2}$ Zentrum für Molekulare Biologie (ZMBH), Universität Heidelberg, Im Neuenheimer Feld 282, D-69120 Heidelberg, Germany
\end{abstract}

Corresponding author: Ban, Nenad (ban@mol.biol.ethz.ch)

\section{Introduction \\ Protein synthesis}

The ribosome carries out protein synthesis in all kingdoms of life by translating genetic information into the amino acid sequences of proteins. In bacteria, the ribosome consists of a large (50S) and a small (30S) subunit, which associate into the active $70 \mathrm{~S}$ ribosome during translation. The small subunit is responsible for decoding the sequence of the mRNA template, whereas the large subunit catalyzes peptide bond formation between amino acids delivered to the active site as amino-acylated tRNAs. During this process, amino acids are attached to the $\mathrm{C}$ terminus of the growing chain with a speed of approximately 20 residues per second in bacteria. The nascent peptide leaves the ribosome through a $100 \AA$ long exit tunnel in the large subunit, which accommodates about 35 amino acids. Its diameter of approximately $15 \AA$ prohibits complete folding of protein domains. Still, a helix-like state has recently been observed for integral inner membrane proteins early in the tunnel $\left[1^{\bullet}\right]$ and further compaction inside the tunnel has been suggested based on cryo-EM imaging of stalled ribosomes [2]. Nevertheless, while the C-terminal part of the protein (or of an autonomously folding domain for multidomain proteins) is still being synthesized in the active site of the ribosome, the $\mathrm{N}$ terminus of the nascent protein leaves the ribosomal tunnel in a mostly unfolded state. Consequently, the folding information generated during biosynthesis is incomplete, and therefore precise timing between synthesis and co- or post-translational folding events is required to avoid irreversible misfolding, proteolysis and aggregation. Therefore, all cells contain a network of chaperones that control the folding process spatially and temporally, thereby minimizing misfolding and premature degradation and promoting efficient folding of proteins.

\section{Bacterial chaperones}

Bacteria contain a large repertoire of cytosolic chaperones with partially overlapping functions. Among those, three chaperones are known to be crucial for de novo folding in Escherichia coli [3,4] (Figure 1). The ribosome-associated chaperone trigger factor (TF) is the first to interact with nascent chains. DnaK, together with its co-chaperone DnaJ and the nucleotide exchange factor GrpE, assists protein folding either co- or post-translationally, whereas the GroEL-GroES chaperonin acts mainly post-translationally. In contrast to the GroEL and DnaK systems, TF is ATP independent and does not require a co-chaperone. Deletion of TF does not affect the growth of $E$. coli, because it can be compensated for by increased activities of DnaK and GroEL [5,6]. However, the combined knockout of TF and DnaK causes massive protein aggregation and cell death at temperatures above $30{ }^{\circ} \mathrm{C}[5,6]$. Whereas the DnaK system is well understood [7], the mechanism of TF has, until recently, remained enigmatic, in particular due to the lack of structural information.

At least $10 \%$ of all bacterial proteins do not remain in the cytosol for folding, but are translocated through or inserted into the membrane in an unfolded state. This secretion is mediated by bacterial signal recognition particle (SRP) and Sec proteins that interact with signal sequence containing nascent chains during or soon after their ribosomal biosynthesis. As the first protein to interact with nascent chains, TF has the potential to regulate 


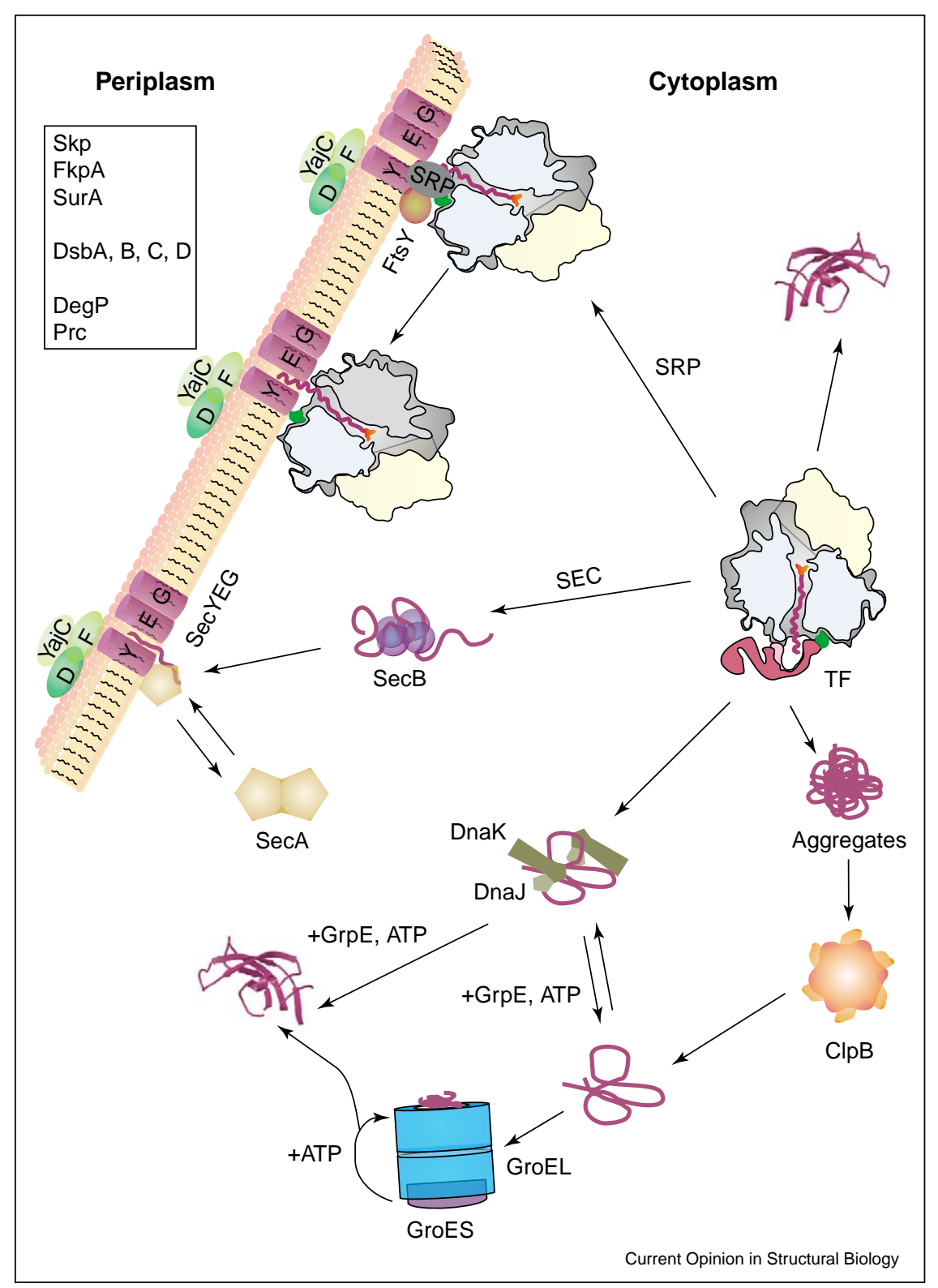

Overview of protein folding and targeting in bacteria. Nascent chains first interact with the ribosome-associated chaperone TF. Depending on their future function, proteins either fold inside the cytosol or are exported through or inserted into the inner membrane. In the cytosol, proteins might fold without further assistance or their folding may be facilitated by additional chaperones, mainly DnaK with its co-chaperone DnaJ and the GroEL-GroES chaperonin. ClpB breaks apart protein aggregates (e.g. those induced by heat stress). The export pathway through the SecYEG translocon can be accessed via two routes. In the first, integral membrane proteins carrying a hydrophobic $\mathrm{N}$-terminal signal anchor sequence are inserted into the inner membrane. This pathway strictly depends on the early co-translational recognition of the signal anchor sequence by SRP (Ffh, 4.5S RNA) and subsequent targeting by the SRP receptor homologue FtsY to the translocon. Upon productive docking of the ribosome onto the translocon and release of SRP and FtsY, proteins are co-translationally inserted into the inner membrane. The second pathway is for the secretion of periplasmic and OMPs; these have less hydrophobic secretion signal sequences and are not recognized by SRP. Instead, these proteins interact, together with cytosolic proteins, during the late co-translational phase or post-translationally with $\mathrm{SecB}$, which keeps them in a translocation-competent non-native state. SecB then selectively transfers secretory proteins by direct interaction to SecA, which shuttles them to the translocon [50]. In contrast to prior assumptions, SecA is active in substrate association as a monomer, at least in the membrane- and translocon-interacting state. Substrate translocation through the translocon is driven by the SecA ATPase [51]. In the periplasm, an additional arsenal of chaperones welcomes the protein. 
the access of SRPs to N-terminal signal sequences and is capable of keeping emerging polypeptides in a translocation-competent non-native conformation [8,9]. Interestingly, the non-folded state of proteins secreted through the membrane into the periplasm via the translocon resembles that of cytosolic proteins emerging at the exit of the ribosomal tunnel. Consistent with this, the periplasm harbours a specialized subset of chaperones, which have to cover the same tasks as cytosolic chaperones and in addition have to ensure correct disulfide bond formation [8].

\section{Structure and function of trigger factor Trigger factor folds into a unique dragon shape} E. coli $\mathrm{TF}$ is a $48 \mathrm{kDa}$ protein, composed of an N-terminal ribosome-binding (RB) 'tail' domain, a peptidyl-prolyl- cis/trans isomerase (PPIase) 'head' domain and a C-terminal portion without sequence homologues [10-12]. It binds in a $1: 1$ stoichiometry to the $50 \mathrm{~S}$ ribosomal subunit in the vicinity of the exit tunnel [13]. Recently, the crystal structure of TF has been determined at $2.7 \AA$ resolution $\left[14^{\bullet \bullet}\right]$. It adopts an unusual extended fold resembling a crouching dragon, in which, surprisingly, the tertiary structure does not represent the linear domain order (Figure 2). The N-terminal RB domain is connected via a long linker to the PPIase domain at the opposite end of the molecule and the C-terminal domain is inserted in between. The C-terminal domain is composed of two helical 'arms' that extend about $40 \AA$ from the back of the protein in the same direction as the tail. In both a C-terminally truncated TF, in which the second arm is completely disordered $\left[15^{\circ}\right]$, and the isolated $\mathrm{RB}$ and

Figure 2

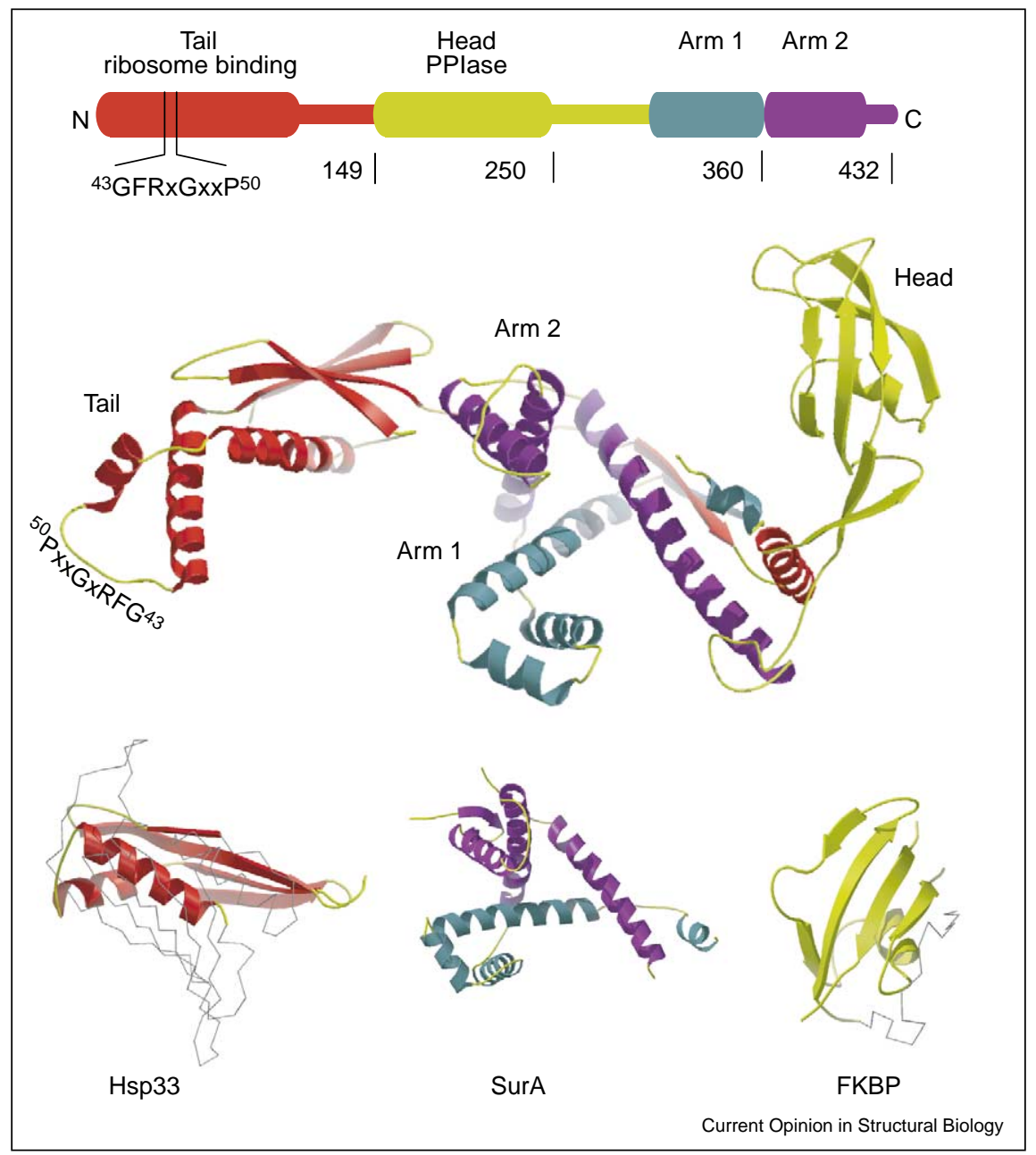

The TF fold exhibits a modular architecture. The figure shows the linear domain organization, fold overview, and comparison to the domains of SurA and Hsp33. The N-terminal RB 'tail' domain (red) is connected via a long linker to the second domain in the linear sequence, which harbours the PPlase activity ('head', yellow). The C-terminal domain, with its two 'arms' (arm 1, green; arm 2, violet), is inserted in between. The loop within the $\mathrm{N}$-terminal domain that is known to be responsible for ribosome binding is marked with the corresponding amino acid sequence. The comparison to the known structures of Hsp33 and SurA, and to the conserved FK506-binding protein (FKBP) fold demonstrates the modular architecture of TF. The corresponding parts of the structure are coloured accordingly. 
PPIase domains, the structures closely resemble the corresponding portions of full-length $\mathrm{TF}$, demonstrating that the unusual open and extended structure of TF is relatively rigid $[16,17]$.

TF has a modular architecture and its domains have structural homology to domains found in other proteins implicated in assisting protein folding (Figure 2). The RB domain is similar to parts of the chaperone Hsp33 [16], although this has no obvious functional relevance for TF. The PPIase domain belongs to the FK506-binding protein family [17], whereas the C-terminal domain shows surprising structural homology to the periplasmic chaperone SurA, as discussed below.

Dimerization of the free TF has been observed in vitro [18]. Nevertheless, it is not clear whether the contacts observed in the crystal structure actually represent physiological dimer interactions. However, the role of TF in the folding of newly synthesized proteins critically depends on its association with the ribosome as a monomer and an in vivo function for the TF dimer remains elusive [13].

\section{A structural model of the ribosome-trigger factor complex}

Concomitantly with the determination of the structure of full-length TF, the crystal structure of the RB domain of $\mathrm{TF}$ in complex with the archaeal Haloarcula marismortui $50 \mathrm{~S}$ ribosomal subunit has been determined [14*0]. According to competition studies, the RB domain is sufficient for wild-type-like ribosome binding [12].
Furthermore, biochemical evidence suggests an identical mode of binding of TF to E. coli and H. marismortui ribosomes, although there are no archaeal homologues of TF $\left[13,14^{\bullet \bullet}\right]$. Consequently, the co-crystal structure serves as a template for the construction of a model of ribosome-bound full-length TF (Figure 3a). TF binds to the ribosome via a small loop within the $\mathrm{N}$-terminal domain adjacent to helix 2, which contains the signature motif 43-GFRxGxxP-50 and is conformationally flexible in free TF $\left[14^{\bullet \bullet}, 16\right]$. The contacting region on the ribosome is the triple junction between domain III of $23 \mathrm{~S}$ rRNA, L23 and L29. In the most prominent interaction, Glu13 of L23 positions the sidechain of Arg45 of TF for interaction with A1501 (A1392 in E. coli) [14*0]. The limited size of the interface is in agreement with the low affinity of TF for vacant ribosomes. In the model, as expected [12], only the N-terminal domain directly contacts the ribosome; the next closest approach is made by the tips of the TF arms. The chaperone hunches over the exit of the ribosomal tunnel and positions a hydrophobic area formed by its tail, back and arms directly above the emerging nascent chain. Surprisingly, this $40 \AA$ deep and $35 \AA$ wide cradle, open on both sides, is large enough to accommodate globular protein domains up to a molecular weight of approximately $15 \mathrm{kDa}\left[14^{\bullet \bullet}\right]$.

The PPIase domain of TF is located far from the ribosomal exit and is unlikely to interact with emerging nascent chains during early stages of protein folding. Indeed, PPIase activity is not essential for the general chaperone function of TF; a TF construct without a PPIase domain complements the synthetic lethality of

Figure 3

(a)

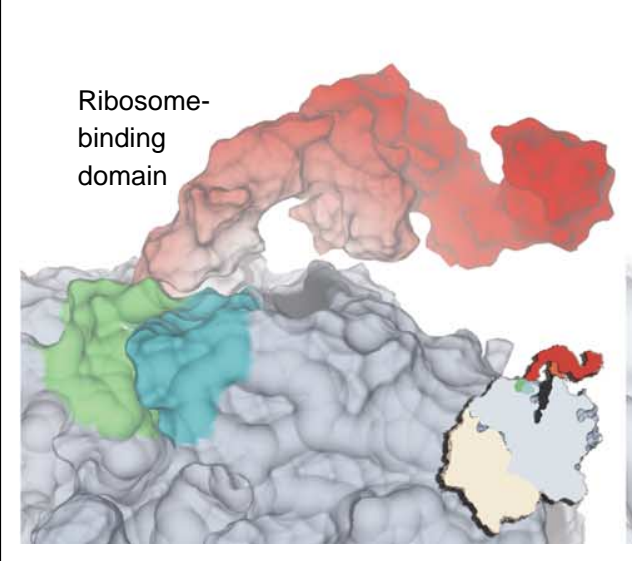

(b)

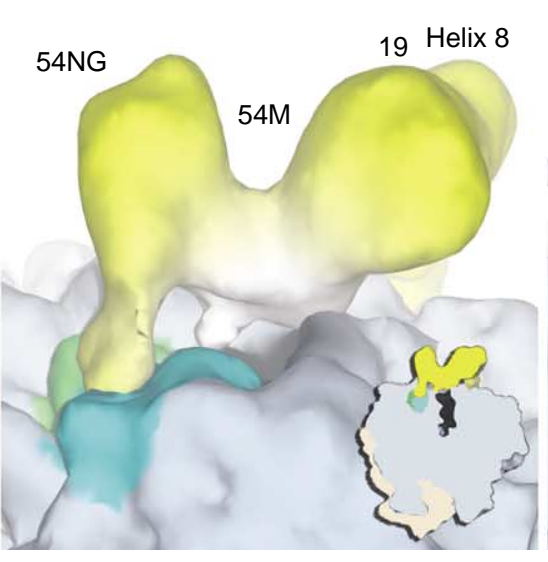

(c)

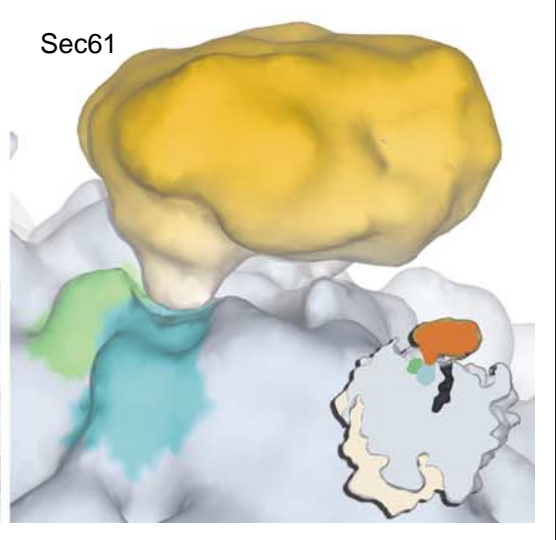

Current Opinion in Structural Biology

Full-length TF bound to the ribosome shown with EM map representations of ribosome-bound SRP and Sec61 translocon. In each panel, a detailed view of the factor bound to the ribosome is shown with an insert displaying a cut-through of the respective complex in identical orientation. The small ribosomal subunit is shown in bisque, the large ribosomal subunit in light blue, ribosomal protein L23 (or its homologues) in light green, L29 (or its homologues) in cyan, TF in red (a), SRP in yellow (b) and Sec61 in orange (c). The ribosomal exit tunnel is coloured black. This comparison illustrates the similar mode of interaction with the ribosome for all three factors, all of which use ribosomal protein L23 as a general docking site. 
combined TF and DnaK deletion mutants [10,19,20]. Nevertheless, it might be required for proper folding of particular, as yet unknown, target proteins [21].

\section{Analogy of cytosolic and periplasmic chaperones?}

Surprisingly, despite a lack of sequence homology, the C-terminal arms of TF resemble the N-terminal chaperone domain of the periplasmic chaperone SurA [22]. Indeed, the analogy of the two proteins extends further: SurA preferentially binds to peptide stretches that are characteristic of outer membrane proteins (OMPs) and are, like the preferred TF-binding sequences, enriched in aromatic residues. SurA also contains two PPIase domains, both of which are not strictly required for SurA function [23,24]. SurA acts in an ATP-independent manner to stabilize secreted OMPs during their passage through the periplasm. SurA null strains are viable, but are characterized by outer membrane perturbations, whereas double knockouts of SurA and either of the periplasmic chaperones DegP or Skp exhibit synthetic lethality $[25,26]$. These chaperones might act at different stages of OMP maturation, but with partially redundant functionality [26]; this is potentially analogous to the cytosolic TF, DnaK, GroEL system.

\section{Co-translational protein folding in prokaryotes}

In vivo experiments demonstrated that co-translational protein folding can occur in the cytosol of both prokaryotic and eukaryotic cells $[27,28]$. However, the absolute proportion of co-translationally folding proteins remains unclear. In fact, in bacteria, but not in reticulocyte lysate, the folding of several large model proteins occurs mainly post-translationally $\left[28,2^{\circ}\right]$, suggesting that the eukaryotic system has a greater capacity for efficient co-translational folding of multidomain proteins than the bacterial one. Perhaps the high speed of bacterial translation per se disfavours co-translational folding [28]. Still, the preferred folding mode of a protein may depend on its particular folding kinetics, its domain sizes and the cellular conditions. A plausible hypothesis assumes that TF may effectively coordinate the stepwise co-translational folding of multidomain proteins, and limit unproductive intermolecular and intramolecular interactions during the early folding steps.

\section{A model of the mechanism of trigger factor assisted co-translational folding}

The formation of a 1:1 complex between TF and vacant ribosomes is required for the interaction of $\mathrm{TF}$ with nascent chains $\left[13,18,30^{\circ}\right]$. In vivo, TF is present at a threefold molar excess over ribosomes and occupies $90 \%$ of them; the non-ribosome-bound fraction of TF is in a monomer-dimer equilibrium [18]. Soon after onset of translation, the interaction with the emerging nascent chain increases the affinity of $\mathrm{TF}$ for the ribosomenascent chain complex (RNC) [11,31]. During ongoing translation, $\mathrm{TF}$ provides shielding of the emerging peptide (Figure 4, step i). It stabilizes unfolded nascent chains and prevents unproductive intramolecular interactions by binding peptide stretches enriched in basic and aromatic residues with its hydrophobic cradle [10,32]. The binding of TF to unfolded proteins decelerates

\section{Figure 4}

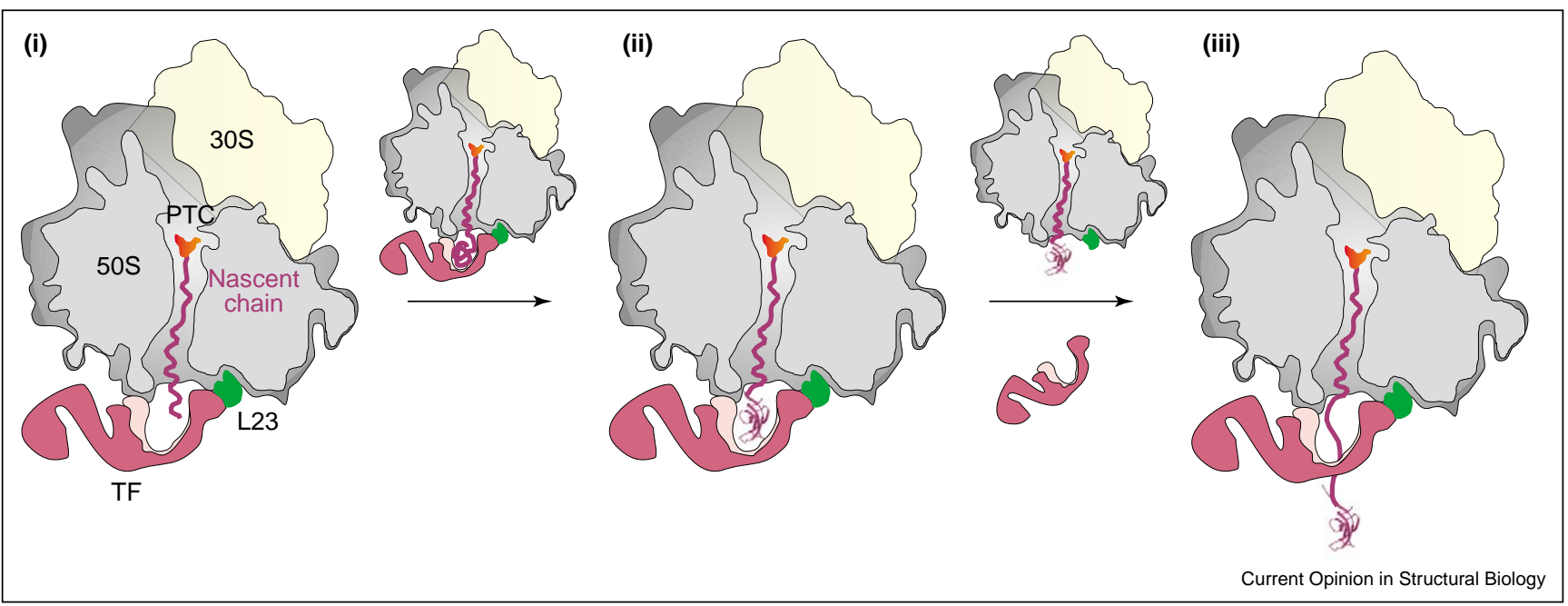

Schematic representation of the model of the mechanism of TF-assisted co-translational protein folding. The small ribosomal subunit is shown in bisque, the large ribosomal subunit in grey. The peptidyl transferase centre (PTC) is indicated in orange, ribosomal protein L23 in green and TF in pink. TF provides a shielded environment for the emerging nascent chain. (i) Its affinity for the RNC is enhanced by the presence of an emerging protein exposing hydrophobic residues. (ii) Once sufficient sequence information is available, the first domain of the growing polypeptide adopts its native fold. During the folding process, hydrophobic residues become buried inside the folded domain; consequently, TF looses affinity for the RNC and dissociates from the ribosome. (iii) In the case of multidomain proteins, TF can rebind after synthesis of further parts of the protein to assist the folding of the second domain. 
the folding process, as confirmed by in vitro refolding experiments [33]. Small proteins or domains might adopt their native fold within the TF cradle, resulting in burial of hydrophobic residues and subsequent destabilization and dissociation of the RNC-TF complex (Figure 4, step ii). In the case of multidomain proteins, $\mathrm{TF}$ rebinding would be favoured upon appearance of newly synthesized unfolded portions with exposed hydrophobic residues (Figure 4, step iii). Protein domains larger than the size of the cradle must either escape from the cradle or promote dissociation of $\mathrm{TF}$ from the ribosome before productive folding. Although the stable association of TF with short nascent chains after their induced release from the ribosome has not been observed [11], increasing amounts of TF are recruited to RNCs during the production of multidomain firefly luciferase $\left[29^{\circ}\right]$, indicating that the ongoing translation of a large multidomain protein indeed expels nascent-chain-bound TF from the ribosome.

\section{Interplay of trigger factor and DnaK}

Mediated by its co-chaperone DnaJ, DnaK binds in an ATP-dependent manner to unfolded proteins with hydrophobic stretches flanked by basic amino acid residues. Its binding specificity overlaps with that of TF $[34,35]$ and they share a substrate pool of about 340 proteins in vivo [36]. In contrast to TF, DnaK is not localized on the ribosome and mainly associates with newly synthesized proteins post-translationally, except for some longer ribosome-bound nascent chains, preferentially larger than 20 $\mathrm{kDa}[5,6,11,37]$. In total, only $10-15 \%$ of all newly synthesized proteins interact transiently with DnaK in vivo. In TF-deficient strains, this number increases to $40 \%$ and DnaK uncharacteristically also interacts with shorter nascent chains $[5,6,20]$. A competitive advantage for TF over DnaK in binding to short nascent chains has also been demonstrated directly by cross-linking studies [36]. Recently, it has been shown that TF and DnaK are required for efficient folding of a multidomain model protein during in vitro translation and cooperatively decrease the folding speed of the studied proteins, thereby shifting folding towards a post-translational mode. However, it remains unclear whether these observations represent a general rule or are specific to the studied proteins $\left[2^{\circ}\right]$. With regard to the model of TF action, the main function of DnaK is seemingly to assist the folding of larger substrates, which can't be accommodated in the TF cradle.

\section{Trigger factor and the secretory pathway}

$\mathrm{TF}$ is the first protein to interact with almost all nascent chains because, under physiological conditions, it is bound to most ribosomes in the cell. Due to the spatial and temporal overlap of its action with the early events in protein targeting, TF combines two contrary roles. On the one hand, it provides shielding of nascent polypeptides in the space defined by its cradle and the widening riboso- mal tunnel. On the other hand, it has to provide access to the nascent chain, such that periplasmic and membrane proteins are recognized early enough to be targeted to the translocon by the SRP- or Sec-dependent pathways in a translocation-competent unfolded state, as outlined in Figure 1. TF has no involvement in post-translational Tat-mediated export beyond its general chaperone function [38].

\section{L23 constitutes a general docking site on the ribosome}

The crystal structure of the RB domain-ribosome complex identified, in agreement with cross-linking studies $[13,39]$, protein L23 as the main interaction site with TF $\left[14^{\bullet \bullet}\right]$. Remarkably, the same protein participates in contacts with ribosome-associated factors involved in protein export. The eukaryotic homologue of L23 mediates a prominent contact with SRP54 [40 ${ }^{\bullet \bullet}$, the subunit of the SRP that is responsible for nascent chain binding (Figure 3b), suggesting an identical mode of interaction for its bacterial counterpart, Ffh. Based on a $15 \AA$ cryoEM reconstruction, $\mathrm{L} 23$ also mediates a major interaction of the eukaryotic translocon, Sec61, with the ribosome (Figure 3c) [41]. Such contacts have also been observed for the bacterial translocon SecYEG-RNC complex (C Schaffitzel, personal communication). The importance of L23 for the docking of protein export factors even extends to the structurally distinct $55 \mathrm{~S}$ mitochondrial ribosome [42]. Here, the Oxa1 translocase, which facilitates protein insertion into the inner mitochondrial membrane, efficiently cross-links to the mitochondrial L23 homologue [43].

\section{SRP-dependent export}

Surprisingly, simultaneous binding of SRP and TF to the ribosome has been observed in spite of the fact that they both use L23 as a contact site and occupy sterically exclusive regions on the ribosomal surface [44,45 ${ }^{\circ}$. These observations suggest that conformational rearrangements must take place to permit binding of both factors at the same time. Biochemical data further suggest that binding of FtsY, the bacterial SRP receptor homologue, to the SRP-TF-ribosome complex excludes the TF [45 ${ }^{\circ}$. SRP exhibits high on/off rates, enabling it to sample all ribosomes for signal anchor sequences, although it is present at substoichiometric amounts in vivo $\left[45^{\circ}\right]$. Competition between TF and SRP for binding to signal anchor sequences has been demonstrated by cross-linking studies, indicating a clear advantage for SRP over TF [39]. However, the presence of nascent-chain-interacting TF prevents the binding of SRP to the less hydrophobic signal sequences of proteins destined for Sec-dependent export and thus increases the specificity of SRP for its cognate substrates $[9,46]$.

\section{Sec-dependent export}

Deletion of TF accelerates Sec-dependent secretion and reduces the requirement for $\mathrm{SecB}$ as a targeting factor, 
such that translocation occurs even in its absence and perhaps already in a co-translational rather than in a posttranslational mode. Based on these observations and the fact that TF has primary access to nascent chains, the probable function of $\mathrm{SecB}$ in translocation is to receive polypeptides from $\mathrm{TF}$ and keep them in an unfolded translocation-competent state for subsequent post-translational transport through the membrane [47]. The transfer of nascent chains might be guided by the occurrence of low-affinity binding of SecB to free TF in solution [48]. The general ability of SecB to prevent the premature folding of nascent chains has recently become evident from the fact that the tenfold overexpression of already abundant SecB can suppress the growth defects of a strain lacking TF and DnaK. Its co-translational binding to short nascent cytosolic and secretory proteins is indiscriminately suppressed by the presence of TF [49 ${ }^{\circ}$. However, its role in protein translocation and the strong overexpression of $\mathrm{SecB}$ required for suppression of the TF-/DnaK- phenotype indicate that it doesn't promote folding as efficiently as the combination of $\mathrm{TF}$ and DnaK.

\section{Conclusions}

To fulfil its role in the early co-translational phase of protein folding, $\mathrm{TF}$ requires two apparently conflicting properties: it has to interact with and shield nascent chains to facilitate co-translational folding, while also permitting and potentially controlling the access of other factors to the nascent chains. The unique dragon-shaped fold of TF seems perfectly tailored to match both requirements. On one hand, the cradle has a hydrophobic inner surface, which enables TF to interact with hydrophobic patches of nascent chains, thus preventing them from getting trapped in unproductive folding intermediates. On the other hand, TF, due to its unusual open shape and relatively weak affinity for the ribosome (mediated by delicate contacts), is in a position to precisely control the handover of nascent chains to downstream post-translational folding factors, such as DnaK, SecB and GroEL/ES, or to the co-translational protein targeting machinery. Remarkably, even in the presence of TF, bacterial SRP is able to gain access to the nascent chain and bind to hydrophobic signal anchor sequences. The competition between TF and SRP for binding to less hydrophobic signal secretion sequences enhances the specificity of SRP. TF and SRP, together with additional factors involved in protein secretion (e.g. the translocon and the mitochondrial Oxa1 protein), utilize ribosomal protein L23 (or its homologues) as a general interaction platform.

Molecular details of the interplay between TF and posttranslational folding factors, and between $\mathrm{TF}$ and components of the co-translational protein translocation machinery remain to be elucidated. Significant contributions can be expected from interdisciplinary structural studies of the individual complexes involved in these processes.

\section{Acknowledgements}

We gratefully acknowledge support from the Swiss National Science Foundation (SNSF), NCCR Structural Biology of the SNSF, ETH research grant TH-3/04-1, a grant from the Deutsche

Forschungsgemeinschaft to ED, and a Young Investigator Grant from the Human Frontier Science Program to NB and ED.

\section{References and recommended reading}

Papers of particular interest, published within the annual period of review, have been highlighted as:

- of special interest

$\bullet$ of outstanding interest

1. Woolhead CA, McCormick PJ, Johnson AE: Nascent membrane - $\quad$ and secretory proteins differ in FRET-detected folding far inside the ribosome and in their exposure to ribosomal proteins. Cell 2004, 116:725-736.

This work demonstrates helix-like protein folding inside the ribosomal exit tunnel, observed only for membrane proteins. This early compaction may play a decisive role regarding the fate of the protein, and its future interactions with TF and SRP.

2. Gilbert RJ, Fucini $P$, Connell S, Fuller SD, Nierhaus $\mathrm{KH}$, Robinson CV, Dobson CM, Stuart DI: Three-dimensional structures of translating ribosomes by cryo-EM. Mol Cell 2004, 14:57-66.

3. Bukau B, Deuerling E, Pfund C, Craig EA: Getting newly synthesized proteins into shape. Cell 2000, 101:119-122.

4. Hartl FU, Hayer-Hartl M: Molecular chaperones in the cytosol: from nascent chain to folded protein. Science 2002, 295:1852-1858.

5. Deuerling E, Schulze-Specking A, Tomoyasu T, Mogk A, Bukau B: Trigger factor and DnaK cooperate in folding of newly synthesized proteins. Nature 1999, 400:693-696.

6. Teter SA, Houry WA, Ang D, Tradler T, Rockabrand D, Fischer G, Blum P, Georgopoulos C, Hartl FU: Polypeptide flux through bacterial Hsp70: DnaK cooperates with trigger factor in chaperoning nascent chains. Cell 1999 97:755-765.

7. Bukau B, Horwich AL: The Hsp70 and Hsp60 chaperone machines. Cell 1998, 92:351-366.

8. Muller M, Koch HG, Beck K, Schafer U: Protein traffic in bacteria: multiple routes from the ribosome to and across the membrane. Prog Nucleic Acid Res Mol Biol 2001, 66:107-157.

9. Beck K, Wu LF, Brunner J, Muller M: Discrimination between SRP- and SecA/SecB-dependent substrates involves selective recognition of nascent chains by SRP and trigger factor. $E M B O J$ 2000, 19:134-143.

10. Kramer G, Rutkowska A, Wegrzyn RD, Patzelt H, Kurz TA, Merz F, Rauch T, Vorderwulbecke S, Deuerling E, Bukau B: Functional dissection of Escherichia coli trigger factor: unraveling the function of individual domains. $J$ Bacteriol 2004, 186:3777-3784.

11. Hesterkamp T, Hauser S, Lutcke H, Bukau B: Escherichia coli trigger factor is a prolyl isomerase that associates with nascent polypeptide chains. Proc Natl Acad Sci USA 1996, 93:4437-4441

12. Hesterkamp T, Deuerling E, Bukau B: The amino-terminal 118 amino acids of Escherichia coli trigger factor constitute a domain that is necessary and sufficient for binding to ribosomes. J Biol Chem 1997, 272:21865-21871.

13. Kramer G, Rauch T, Rist W, Vorderwulbecke S, Patzelt H, Schulze-Specking A, Ban N, Deuerling E, Bukau B: L23 protein functions as a chaperone docking site on the ribosome. Nature 2002, 419:171-174. 
14. Ferbitz L, Maier T, Patzelt H, Bukau B, Deuerling E, Ban N:

- Trigger factor in complex with the ribosome forms a molecular cradle for nascent proteins. Nature 2004, 431:590-596.

This article describes X-ray crystal structures of full-length TF at $2.7 \AA$ resolution and of the RB domain of TF in complex with the $50 \mathrm{~S}$ ribosomal subunit at 3.5 A resolution. It also provides a structure-based model of TF action.

15. Ludlam AV, Moore BA, Xu Z: The crystal structure of ribosomal - $\quad$ chaperone trigger factor from Vibrio cholerae. Proc Natl Acad Sci USA 2004, 101:13436-13441.

Ludlam et al. report the crystal structure of a C-terminally truncated form of Vibrio cholerae TF, in which the second arm is completely disordered. The remainder of the molecule adopts a similar conformation as in fulllength $E$. coli TF $\left[14^{\circ}{ }^{\circ}\right]$, demonstrating the rigidity of the overall TF fold.

16. Kristensen $\mathrm{O}$, Gajhede M: Chaperone binding at the ribosomal exit tunnel. Structure 2003, 11:1547-1556.

17. Vogtherr M, Jacobs DM, Parac TN, Maurer M, Pahl A, Saxena K, Ruterjans $H$, Griesinger $C$, Fiebig KM: NMR solution structure and dynamics of the peptidyl-prolyl cis-trans isomerase domain of the trigger factor from Mycoplasma genitalium compared to FK506-binding protein. J Mol Biol 2002, 318:1097-1115.

18. Patzelt H, Kramer G, Rauch T, Schonfeld HJ, Bukau B, Deuerling E: Three-state equilibrium of Escherichia coli trigger factor. Biol Chem 2002, 383:1611-1619.

19. Kramer G, Patzelt H, Rauch T, Kurz TA, Vorderwulbecke S, Bukau B, Deuerling E: Trigger factor peptidyl-prolyl cis/trans isomerase activity is not essential for the folding of cytosolic proteins in Escherichia coli. $J$ Biol Chem 2004, 279:14165-14170.

20. Genevaux P, Keppel F, Schwager F, Langendijk-Genevaux PS, Hartl FU, Georgopoulos C: In vivo analysis of the overlapping functions of DnaK and trigger factor. EMBO Rep 2004, 5:195-200.

21. Lyon WR, Caparon MG: Trigger factor-mediated prolyl isomerization influences maturation of the Streptococcus pyogenes cysteine protease. J Bacteriol 2003, 185:3661-3667.

22. Bitto E, McKay DB: Crystallographic structure of SurA, a molecular chaperone that facilitates folding of outer membrane porins. Structure 2002, 10:1489-1498.

23. Behrens $S$, Maier R, de Cock H, Schmid FX, Gross CA: The SurA periplasmic PPlase lacking its parvulin domains functions in vivo and has chaperone activity. $E M B O J \mathrm{~J} 2001$, 20:285-294.

24. Bitto E, McKay DB: Binding of phage-display-selected peptides to the periplasmic chaperone protein SurA mimics binding of unfolded outer membrane proteins. FEBS Lett 2004, 568:94-98.

25. Krojer T, Garrido-Franco M, Huber R, Ehrmann M, Clausen T: Crystal structure of DegP (HtrA) reveals a new protease-chaperone machine. Nature 2002, 416:455-459.

26. Rizzitello AE, Harper JR, Silhavy TJ: Genetic evidence for parallel pathways of chaperone activity in the periplasm of Escherichia coli. J Bacteriol 2001, 183:6794-6800.

27. Nicola AV, Chen W, Helenius A: Co-translational folding of an alphavirus capsid protein in the cytosol of living cells. Nat Cell Biol 1999, 1:341-345.

28. Netzer WJ, Hartl FU: Recombination of protein domains facilitated by co-translational folding in eukaryotes. Nature 1997, 388:343-349.

29. Agashe VR, Guha S, Chang HC, Genevaux P, Hayer-Hartl M,

- Stemp M, Georgopoulos C, Hartl FU, Barral JM: Function of trigger factor and DnaK in multidomain protein folding: increase in yield at the expense of folding speed. Cell 2004, 117:199-209.

The authors provide a detailed analysis of the influence of DnaK and TF on the folding kinetics of two model proteins, $\beta$-galactosidase and firefly luciferase, during in vitro translation.

30. Maier R, Eckert B, Scholz C, Lilie H, Schmid FX: Interaction

- of trigger factor with the ribosome. J Mol Biol 2003, 326:585-592.
A detailed investigation of the dynamics of the TF-ribosome interaction, using a TF variant with a fluorescent label attached to its $\mathrm{N}$-terminal $\mathrm{RB}$ domain. It revealed that the TF-ribosome complex has much longer lifetimes than complexes between TF and artificial unfolded protein substrates without the context of the ribosome.

31. Valent QA, de Gier JW, von Heijne G, Kendall DA, ten Hagen-Jongman CM, Oudega B, Luirink J: Nascent membrane and presecretory proteins synthesized in Escherichia coli associate with signal recognition particle and trigger factor. Mol Microbiol 1997, 25:53-64.

32. Patzelt H, Rudiger $\mathrm{S}$, Brehmer D, Kramer G, Vorderwulbecke $S$, Schaffitzel E, Waitz A, Hesterkamp T, Dong L, SchneiderMergener $\mathrm{J}$ et al.: Binding specificity of Escherichia coli trigger factor. Proc Natl Acad Sci USA 2001, 98:14244-14249.

33. Maier R, Scholz C, Schmid FX: Dynamic association of trigger factor with protein substrates. $J$ Mol Biol 2001, 314:1181-1190.

34. Rudiger S, Germeroth L, Schneider-Mergener J, Bukau B: Substrate specificity of the DnaK chaperone determined by screening cellulose-bound peptide libraries. EMBO J 1997, 16:1501-1507.

35. Rudiger S, Schneider-Mergener J, Bukau B: Its substrate specificity characterizes the DnaJ co-chaperone as a scanning factor for the DnaK chaperone. EMBO J 2001, 20:1042-1050.

36. Deuerling $E$, Patzelt $H$, Vorderwulbecke $S$, Rauch $T$, Kramer $G$ Schaffitzel E, Mogk A, Schulze-Specking A, Langen H, Bukau B: Trigger factor and DnaK possess overlapping substrate pools and binding specificities. Mol Microbiol 2003, 47:1317-1328.

37. Kramer G, Ramachandiran V, Horowitz PM, Hardesty B: The molecular chaperone DnaK is not recruited to translating ribosomes that lack trigger factor. Arch Biochem Biophys 2002, 403:63-70.

38. Jong WS, Ten Hagen-Jongman CM, Genevaux $\mathrm{P}$, Brunner J, Oudega B, Luirink J: Trigger factor interacts with the signal peptide of nascent Tat substrates but does not play a critical role in Tat-mediated export. Eur J Biochem 2004, 271:4779-4787.

39. Ullers RS, Houben EN, Raine A, ten Hagen-Jongman CM, Ehrenberg M, Brunner J, Oudega B, Harms N, Luirink J: Interplay of signal recognition particle and trigger factor at L23 near the nascent chain exit site on the Escherichia coli ribosome. J Cell Biol 2003, 161:679-684.

40. Halic M, Becker T, Pool MR, Spahn CM, Grassucci RA,

- Frank J, Beckmann R: Structure of the signal recognition particle interacting with the elongation-arrested ribosome. Nature 2004, 427:808-814.

Cryo-EM reconstruction of the eukaryotic ribosome-SRP complex and model building of the complex based on existing crystal structures provide a comprehensive view of ribosome-SRP and SRP-nascent chain interactions.

41. Beckmann R, Spahn CM, Eswar N, Helmers J, Penczek PA, Sali A, Frank J, Blobel G: Architecture of the protein-conducting channel associated with the translating $80 \mathrm{~S}$ ribosome. Cell 2001, 107:361-372.

42. Sharma MR, Koc EC, Datta PP, Booth TM, Spremulli LL, Agrawal RK: Structure of the mammalian mitochondria ribosome reveals an expanded functional role for its component proteins. Cell 2003, 115:97-108.

43. Jia L, Dienhart M, Schramp M, McCauley M, Hell K, Stuart RA: Yeast Oxa1 interacts with mitochondrial ribosomes: the importance of the C-terminal region of Oxa1. EMBO J 2003, 22:6438-6447.

44. Raine A, Ivanova N, Wikberg JE, Ehrenberg M: Simultaneous binding of trigger factor and signal recognition particle to the E. coli ribosome. Biochimie 2004, 86:495-500.

45. Buskiewicz I, Deuerling E, Gu SQ, Jockel J, Rodnina MV,

- Bukau B, Wintermeyer W: Trigger factor binds to ribosomesignal-recognition particle (SRP) complexes and is excluded by binding of the SRP receptor. Proc Natl Acad Sci USA 2004, 101:7902-7906. 
The authors present an analysis of the interactions between SRP, SRP receptor and TF based on cross-linking patterns and spectroscopical measurements. Unexpectedly, SRP and TF bind to the ribosome simultaneously, and TF dissociates from the complex only after formation of the TF-SRP-SRP receptor-ribosome quaternary complex.

46. Lee HC, Bernstein HD: The targeting pathway of Escherichia coli presecretory and integral membrane proteins is specified by the hydrophobicity of the targeting signal. Proc Natl Acad Sci USA 2001, 98:3471-3476.

47. Lee HC, Bernstein HD: Trigger factor retards protein export in Escherichia coli. J Biol Chem 2002, 277:43527-43535.

48. Ha SC, Lee TH, Cha SS, Kim KK: Functional identification of the SecB homologue in Methanococcus jannaschii and direct interaction of SecB with trigger factor. Biochem Biophys Res Commun 2004, 315:1039-1044.
49. Ullers RS, Luirink J, Harms N, Schwager F, Georgopoulos C,

- Genevaux P: SecB is a bona fide generalized chaperone in Escherichia coli. Proc Natl Acad Sci USA 2004, 101:7583-7588.

This study demonstrates that $\mathrm{SecB}$ overexpression efficiently suppresses the synthetic lethality of an E. coli strain lacking both TF and DnaK, and that $\operatorname{SecB}$ interacts with nascent chains of secreted and also non-secreted proteins in the absence of TF. Based on these observations, the authors suggest a generalized role for SecB in protein folding.

50. Fisher AC, DeLisa MP: A little help from my friends: quality control of presecretory proteins in bacteria. $J$ Bacteriol 2004, 186:7467-7473.

51. Or E, Boyd D, Gon S, Beckwith J, Rapoport T: The bacterial ATPase SecA functions as a monomer in protein translocation. J Biol Chem 2005, 280:9097-9105. 